\title{
Optical bottle vs. acoustic bottle and antibottle resonators
}

\author{
M. SUMETSKY \\ Aston Institute of Photonic Technologies, Aston University, Birmingham B4 7ET, UK \\ Corresponding author: m.sumetsky@aston.ac.uk
}

Received XX Month XXXX; revised XX Month, XXXX; accepted XX Month XXXX; posted XX Month XXXX (Doc. ID XXXXX); published XX Month XXXX

\begin{abstract}
The theory of slow acoustic modes propagating along the optical fiber and controlled by nanoscale variation of the effective fiber radius (analogous to the theory of slow optical whispering gallery modes) is developed. Surprisingly, it is shown that, in addition to acoustic bottle resonators (which are similar to optical bottle resonators), there exist antibottle resonators - the neckshaped deformations of the fiber which can fully confine acoustic modes. It is also shown that an eigenfrequency of mechanical vibrations of silica parabolic bottle resonator can match the separation between eigenfrequencies of series of its optical modes thereby enabling resonant mechanical excitation of these series. The developed theory paves the groundwork for slow mode optomechanics in an optical fiber. (c) 2017 Optical Society of America
\end{abstract}

OCIS codes: (060.2340) Fiber optics components; (140.3945)

Microcavities; (230.3990) Micro-optical devices.

http://dx.doi.org/10.1364/OL.99.099999

Optical bottle resonators (elongated swellings formed along an optical fiber, Fig. 1(a)) introduced more than a decade ago [1] have been explored for applications in cavity quantum electrodynamics [2], all-optical switching [3], frequency comb generation [4, 5], spectral cleaning [6], quantum computing [7], slow light delay lines [8], optomechanics [9], and other topics of photonics. Of special interest are bottle resonators created by nanoscale variation of the effective fiber radius. These resonators, explored in Surface Nanoscale Axial Photonics (SNAP) [10], can confine slow whispering gallery modes (SWGMs) which radiation frequency is close to a cutoff frequency. The propagation constant of SWGMs is very small and can be controlled by nanoscale variation of the effective fiber radius. In contrast to regular spherical, toroidal, and bottle resonators, SNAP bottle resonators can be fabricated with unprecedented precision approaching 0.1 angstrom [11].

Stimulated by the recent progress in optomechanics of microresonators [12], the SNAP platform can be favorably enriched by taking into account the interaction of optical SWGMs with acoustic modes of the fiber, i.e., by the development of SWGM optomechanics. To this end, it is important to find out if, similar to optical SWGMs, there exist slow acoustic modes which can be controlled (and, in particular, fully confined) by nanoscale variation of the effective fiber radius. This Letter gives the positive answer to this question and develops the theory of slow acoustic modes analogous to the SNAP theory.

In contrast to optical modes, the existence of high Q-factor acoustic modes does not require the condition of total internal reflection. For this reason, acoustic SWGMs as well as other slow modes (e.g., axially symmetric modes) can be considered. Surprisingly, it is shown below that, in addition to acoustic bottle resonators (Fig. 1(b)) which are similar to optical bottle resonators (Fig. 1(a)), there exist antibottle resonators - the neck-shaped deformations of the fiber which can fully confine acoustic modes (Fig. 1(c)).

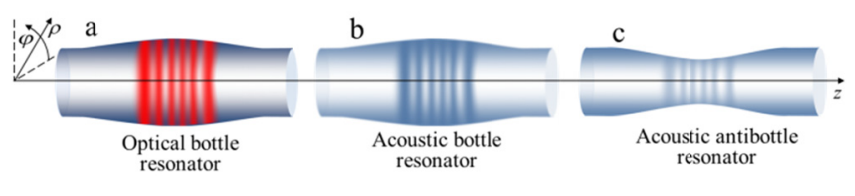

Fig. 1. Illustrations of optical bottle resonator (a), acoustic bottle resonator (b), and acoustic antibottle resonator (c).

It is instructive to start with a brief introduction to the SNAP theory. The electromagnetic field inside a homogeneous fiber satisfies the wave equation [13]

$$
\frac{\partial^{2} \mathbf{U}}{\partial t^{2}}-V^{2}\left[\frac{\partial^{2} \mathbf{U}}{\partial z^{2}}+\frac{1}{\rho} \frac{\partial}{\partial \rho}\left(\rho \frac{\partial \mathbf{U}}{\partial \rho}\right)+\frac{1}{\rho^{2}} \frac{\partial^{2} \mathbf{U}}{\partial \varphi^{2}}\right]=0,
$$

where $V=c / n_{r}$ and $c$ is the velocity of light in the fiber material and vacuum, respectively, and $n_{r}$ is the refractive index of the fiber. Assuming adiabatically slow and small variation of the fiber radius, $r(z)=r_{0}+\Delta r(z)$, we can separate variables in Eq. (1) in the cylindrical frame of reference $(\varphi, \rho, z)$. Solution of Eq. (1) near the cutoff frequency $v_{m, n}$ can be found using the local mode 
approximation [13], $\mathbf{U}_{m, n}(z, \rho, \varphi, t)=\exp (-2 \pi i v t) \Psi_{m, n}(z) \mathbf{Q}_{m, n}(z, \rho, \varphi)$, where $m$ and $n$ are azimuthal and radial quantum numbers and $\Psi_{m, n}(z)$ and $\mathbf{Q}_{m, n}(z, \rho, \varphi)$ are slow functions of axial coordinate $z$ . Function $\mathbf{Q}_{m, n}(z, \rho, \varphi)$ satisfies the local mode equation

$$
\Delta_{\perp} \mathbf{Q}_{m, n}+\left(2 \pi v_{m, n} / V\right)^{2} \mathbf{Q}_{m, n}=0,
$$

where the dependence on $z$ is considered as parametric and the value of propagation constant is set to zero, $\beta_{m, n}(z)=0$. Function $\Psi_{m, n}(z)$ satisfies the one-dimensional wave equation [14]

$$
\frac{\partial^{2} \Psi_{m, n}}{\partial z^{2}}+\beta_{m, n}^{2}(z) \Psi_{m, n}=0
$$

The propagation constant $\beta_{m, n}(z)$ in this equation is expressed through the variation of frequency, $\Delta v=v-v_{m, n}$ and fiber radius $\Delta r(z)$ by expansion of the exact dispersion relation for the cylindrically symmetric uniform optical fiber [13] up to the first order in $\beta_{m, n}^{2}$ [14]:

$$
\begin{gathered}
\beta_{m, n}^{2}(z)=\Lambda_{m, n}\left(\frac{\Delta v}{v}+\frac{\Delta r(z)}{r_{0}}\right), \\
\Lambda_{m, n}=\Lambda_{m, n}^{(o p)}=2\left(k_{m, n}^{(o p)}\right)^{2}, \quad k_{m, n}^{(o p)}=2 \pi v_{m, n}^{(o p)} n_{r} / c,
\end{gathered}
$$

and $k_{m, n}^{(o p)}$ is the propagation constant in the fiber material corresponding to optical cutoff frequency $v_{m, n}^{(o p)}$. The structure of propagation constant determined by Eq. (4) makes Eq. (3) identical to the stationary Schrödinger equation where $-\Delta r(z)$ and $\Delta v$ plays the role of potential and energy, respectively. It will be shown below that Eqs. (3) and (4) describe the axial distribution of acoustic modes as well. To this end, the value of $\Lambda_{m, n}=\Lambda_{m, n}^{(o p)}$ given by Eq. (5) should be replaced by $\Lambda_{m, n}=\Lambda_{m, n}^{(a c)}$ from Eq. (12).

The bottle resonator of our particular interest is the parabolic bottle resonator with

$$
\Delta r(z)=z^{2} /\left(2 R_{0}\right) .
$$

From Eqs. (3)-(6), the axial distribution of SWGMs in this resonator, $\Psi_{m, n}(z)$, satisfies the parabolic cylinder function equation. Since $\quad \Lambda_{m, n}=2\left(k_{m, n}^{(o p)}\right)^{2}$ is always positive, the localized states correspond to negative axial radius, $R_{0}<0$. Their axial distribution is expressed through the Hermite polynomials:

$$
\Psi_{m, n, q}(z)=\exp \left(-\frac{z^{2}}{2 \zeta_{m, n}^{2}}\right) H_{q}\left(\frac{z}{\zeta_{m, n}}\right), \quad \zeta_{m, n}=\left(-\frac{r_{0} R_{0}}{\Lambda_{m, n}}\right)^{1 / 4} .
$$

Here $q$ is the axial quantum number. Analogous to the known result (see e.g., [5]), the eigenfrequencies of the parabolic bottle resonator are determined as

$$
v_{m, n, q}=v_{m, n}\left[1+(2 \pi)^{-1}\left(-\Lambda_{m, n} r_{0} R_{0}\right)^{-1 / 2}\left(q+\frac{1}{2}\right)\right] .
$$

From Eq. (5) this equation is simplified as $v_{m, n, q}=v_{m, n}+c\left(2 \pi n_{r}\right)^{-1}\left(-r_{0} R_{0}\right)^{-1 / 2}\left(q+\frac{1}{2}\right)$.

In the case of acoustic modes, the local displacement of the fiber, $\mathbf{U}_{m, n}$ (denoted here identically to the electromagnetic field discussed above) can be expressed as a sum of longitudinal and transverse modes, $\mathbf{U}_{m, n}^{(L)}$ and $\mathbf{U}_{m, n}^{(T)}$, which satisfy the wave equation, Eq. (1). In this equation, the velocity $V$ should be replaced by the longitudinal sound velocity $V_{L}$ for $\mathbf{U}_{m, n}^{(L)}$ and transverse sound velocity $V_{T}$ for $\mathbf{U}_{m, n}^{(T)}$. The total displacement is found by applying the boundary condition for tension to the sum $\mathbf{U}_{m, n}=\mathbf{U}_{m, n}^{(L)}+\mathbf{U}_{m, n}^{(T)}$ at the fiber surface [15]. Similar to optical modes, for small and adiabatically slow $\Delta r(z)$ we can determine $\mathbf{U}_{m, n}^{(L)}$ and $\mathbf{U}_{m, n}^{(T)}$ in the form $\mathbf{U}_{m, n}^{(L, T)}=\exp (-2 \pi i v t) \Psi_{m, n} \mathbf{Q}_{m, n}^{(L, T)}$ where $\Psi_{m, n}(z)$ satisfies Eq. (3) with the expression for $\beta_{m, n}^{2}(z)$ presented below and $\mathbf{Q}_{m, n}^{(L, T)}$ satisfies Eq. (2) where $V$ is replaced by $V_{L, T}$. Hereafter we restrict the consideration to axially symmetric acoustic modes corresponding to $m=0$ [16], which dispersion relation is determined by the Pochhammer-Chree equation $[17,18]$ :

$$
\begin{aligned}
& {\left[2 \beta^{2}-\left(2 \pi v / V_{T}\right)^{2}\right]^{2} \Upsilon(h r)+4 h^{2} \beta^{2} \Upsilon(k r)-2\left(2 \pi v / V_{T}\right)^{2} h^{2}=0,} \\
& h=\left[\alpha^{2} \Omega^{2}-\beta^{2}\right]^{1 / 2}, \quad k=\left[\Omega^{2}-\beta^{2}\right]^{1 / 2},
\end{aligned}
$$

where $\beta$ is the propagation constant, $\Omega=2 \pi v r / V_{T}, \alpha=V_{T} / V_{L}$, $\Upsilon(x)=x J_{0}(x) / J_{1}(x)$, and $J_{n}(x)$ is the Bessel function. The cutoff frequencies of these modes, $v_{0, n}^{(a c)}=\Omega_{n} V_{T} /(2 \pi r)$, are determined by setting $\beta=0$ in Eq. (5):

$$
\Omega_{n} J_{0}\left(\alpha \Omega_{n}\right)-2 \alpha J_{1}\left(\alpha \Omega_{n}\right)=0 .
$$

Near the cutoff, the transverse component of acoustic modes, $\mathbf{U}_{0, n}^{(T)}(z, \rho)$, vanishes and only the longitudinal modes,

$$
\mathbf{U}_{0, n}^{(L)}(z, \rho)=J_{1}\left(\alpha \Omega_{n} \rho / r_{0}\right) \Psi_{0, n}(z),
$$

remain finite. Nevertheless, the vanishing transverse component affects the boundary condition for at the fiber surface. In fact, for frequencies close to the cutoff (i.e., for small propagation constant $\beta=\beta_{0, n}(z)$ ) expansion of Eq. (9) up to the first order in $\beta_{0, n}^{2}(z), \Delta v$, and $\Delta r(z)$ yields Eq. (4) where

$$
\Lambda_{0, n}=\Lambda_{0, n}^{(a c)}=\frac{1}{r_{0}^{2}} \frac{2 \alpha^{2} \Omega_{n}^{2}(\alpha)\left(4-4 \alpha^{2}-\Omega_{n}^{2}(\alpha)\right)}{\left(\Omega_{n}^{2}(\alpha)-12 \alpha^{2}+8 \alpha^{2} \Upsilon\left(\Omega_{n}(\alpha)\right)\right.},
$$

and $\Omega_{n}(\alpha)$ is calculated from Eq. (10). While the radial distribution of acoustic mode determined by Eq. (11) depends on the longitudinal velocity only, the value of $\Lambda_{0, n}^{(a c)}(\alpha)$ determined by Eqs. (10) and (12) depends on both longitudinal and transverse velocities. For $\Delta r(z)=0$, Eqs. (4), (12) can be revealed from the previous analysis of the Pochhammer-Chree equation [17]. 
Crucially, in contrast to the positive value of $\Lambda_{n, m}$ determined by Eq. (5) for optical modes, the value of $\Lambda_{0, n}^{(a c)}$ determined by Eq. (12) can be both positive and negative. Fig. 2 shows the plots of dimensional parameter $\Lambda_{0, n}^{(a c)} r_{0}^{2}$ as a function of ratio of transverse and longitudinal velocities, $\alpha=V_{T} / V_{L}$, for fundamental radial mode LP00 corresponding to $n=0$ and the next three modes LP01, LP02, and LP03, corresponding to $n=1,2$ and 3 , respectively. The ratio $V_{T} / V_{L}$ is considered in the range $0.4-0.7$ corresponding to the characteristic for glasses Poisson ratios $\sigma=\left(1-2 \alpha^{2}\right) /\left[2\left(1-\alpha^{2}\right)\right] \sim 0.1-0.4$ [19].

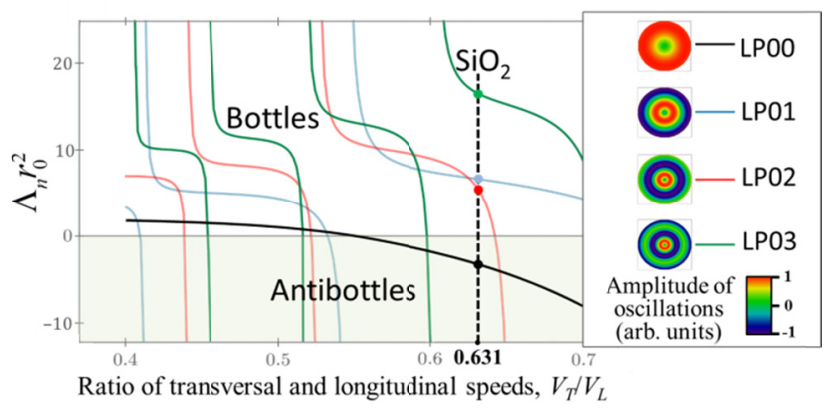

Fig. 2. Dimensionless parameters $\Lambda_{n} r_{0}^{2}$ as a function of $\alpha=V_{T} / V_{L}$ for modes with $n=0,1,2$ and 3 (LP01, LP02, and LP03). The vertical dashed line corresponds to $\alpha=0.631$ for silica. Left: cross-sectional distribution of mode amplitudes for a silica fiber.

Let us now consider the parabolic acoustic bottle resonator with $\Delta r(z)$ defined by Eq. (6). The axial distribution of modes in this resonator and their eigenfrequencies, $v_{0, n, q}=v_{0, n, q}^{(a c)}$, are determined by Eqs. (7) and (8) where $\Lambda_{0, n}=\Lambda_{0, n}^{(a c)}$ (corresponding to $m=0$ ) and cutoff frequencies $v_{0, n}=v_{0, n}^{(a c)}$ determined from Eqs. (10) and (12). Positive values of $\Lambda_{0, n}^{(a c)}$ in Fig. 2 correspond to regular acoustic bottle resonators (Fig. 1(b)) similar to optical bottle resonators (Fig. 1(a)). However, for negative $\Lambda_{0, n}^{(a c)}$ a regular bottle resonator having $R_{0}<0$ (which always confines optical modes) cannot confine acoustic modes. According to Eqs. (7) and (8), the axial distribution of acoustic modes remains the same only if both $\Lambda_{0, n}^{(a c)}$ and $R_{0}$ change sign. Consequently, the condition $\Lambda_{0, n}^{(a c)} R_{0}<0$ corresponds to the full confinement of acoustic modes while for $\Lambda_{0, n}^{(a c)} R_{0}>0$ their spectrum is continuous and axial localization is impossible. Thus, for negative $\Lambda_{0, n}^{(a c)}$ the acoustic modes can be fully confined in a fiber segment having $R_{0}>0$ illustrated in Fig. 1(c). This neck-shaped acoustic resonator is natural to call the antibottle resonator.

Let us consider the important case of a silica fiber having $V_{L}=5970 \mathrm{~m} / \mathrm{s}, V_{T}=3760 \mathrm{~m} / \mathrm{s}, \sigma=0.17$, and $\alpha=0.631$ [20] indicated by the dashed vertical line in Fig. 2. It is seen that in this case the fundamental axial mode LP00 has negative $\Lambda_{0,0}^{(a c)} r_{0}^{2}=-3.266$ while for LP01, LP02, and LP03 the values $\Lambda_{0,1}^{(a c)} r_{0}^{2}=6.638, \Lambda_{0,2}^{(a c)} r_{0}^{2}=5.523$, and $\Lambda_{0,3}^{(a c)} r_{0}^{2}=16.523$ are positive. For comparison, the value of the same parameter of an optical mode calculated from Eq. (5) at characteristic telecommunication frequency $v_{m, n}^{(c u t)}=200 \mathrm{THz}$ and $r_{0}=20 \mu \mathrm{m}$ is $\Lambda_{m, n}^{(o p)} r_{0}^{2} \approx 3 \cdot 10^{4}$. Consequently, the ratio of axial lengths of optical and acoustic modes localized by the same bottle resonator found from Eq. (7) for LP01 is

$$
\zeta_{m, n}^{(o p)} / \zeta_{0,1}^{(a c)}=\left(\Lambda_{0,1}^{(a c)} / \Lambda_{m, n}^{(o p)}\right)^{1 / 4} \approx 0.12 .
$$

Thus, the characteristic axial length of an optical mode is typically an order of magnitude smaller than that of an acoustic mode and slowly increases with the radial number $n$ of acoustic mode as $n^{1 / 4}$ (for example, for $n=50$ we have $\zeta_{m, n}^{(o p)} / \zeta_{0,50}^{(a c)}=0.29$ ).

Of special interest is the case of resonant optomechanical coupling when the acoustic mode eigenfrequency, $v_{0, n, q}^{(a c)}$, is equal to the separation of optical mode eigenfrequencies along the axial quantum number $q$ found from Eq. (8) as $\Delta v^{(o p)}=c\left(2 \pi n_{r}\right)^{-1}\left(-r_{0} R_{0}\right)^{-1 / 2}$. To determine the axial radius of parabolic bottle resonators supporting parametric optomechanical coupling, we consider relatively small acoustic quantum numbers $q$ and replace $v_{0, n, q}^{(a c)}$ by the cutoff frequency $v_{0, n}^{(a c)}=\Omega_{n} V_{T} /\left(2 \pi r_{0}\right)$ so that the condition $\Delta v^{(o p)}=v_{0, n}^{(a c)}$ determines the axial radius as $R_{0, n}=c^{2}\left(\Omega_{n} n_{r} V_{T}\right)^{-2} r_{0}$. For silica fiber with radius $r_{0}=20 \mu \mathrm{m}$ this equation yields $R_{0,0}=6024 \mathrm{~m}, R_{0,1}=824 \mathrm{~m}, R_{0,2}=325 \mathrm{~m}$, $R_{0,3}=173 \mathrm{~m}, R_{0,4}=107 \mathrm{~m}$, decreasing proportionally to $n^{-2}$ for large $n$. As shown in [8] parabolic resonators with such dramatically large axial radii can be introduced experimentally. The acoustic cutoff frequencies corresponding to these radii are $v_{0,0}^{(a c)}=94 \mathrm{MHz}, v_{0,1}^{(a c)}=255 \mathrm{MHz}, \quad v_{0,2}^{(a c)}=406 \mathrm{MHz}, \quad v_{0,3}^{(a c)}=556$ $\mathrm{MHz}, \quad v_{0,4}^{(a c)}=706 \mathrm{MHz}$. These frequencies grow linearly with $n$ for large $n$ and enter the $\mathrm{GHz}$ range similar to the acoustic eigenfrequencies of a ring microresonator experimentally observed in [21]. Similar to the ring resonator, the cross-section of the optical fiber can be structured (e.g., in the form of a capillary or a fiber filled in with other material) to modify its mechanical parameters.

Fig. 3(a) shows the radius variation $\Delta r(z)$ of parabolic bottle resonator with axial radius $R_{0,1}=824 \mathrm{~m}$ corresponding to cutoff acoustic frequency $v_{0,1}^{(a c)}=255 \mathrm{MHz}$ equal to the separation of optical eigenfrequencies $\Delta v^{(o p)}$. The axial distribution of optical modes of this resonator with $q=0,10$ and 100 and its acoustic modes with $q=0,1$ and 2 are shown. The acoustic eigenfrequencies are well defined along the axial quantum number $q$ if their spectral width is much smaller than their separation. In terms of mechanical Q-factor, $Q^{(a c)}$, this condition is written as $Q^{(a c)}>>v_{0, n, q}^{(a c)} / \Delta v_{n}^{(a c)} \quad$ where, from Eq. 
$\Delta v_{n}^{(a c)}=(2 \pi)^{-1}\left(-\Lambda_{m, n} r_{0} R_{0}\right)^{-1 / 2} v_{0, n}$. For acoustic modes shown in Fig. 3(b), we have $v_{0,1, q}^{(a c)} \approx 255 \mathrm{MHz}$ and $\Delta v_{1}^{(a c)}=2.45 \mathrm{KHz}$ so that $Q^{(a c)}>>10^{5}$. The mechanical Q-factor measured at frequencies of our concern is $Q^{(a c)} \leq 10^{5}$ at room temperature [12]. At very low temperatures, $Q^{(a c)}$ of silica grows inverse proportional to the temperature and can achieve values above $10^{6}$ below $0.1 \mathrm{~K}[22,23]$ so that the condition $Q^{(a c)}>10^{5}$ is satisfied. On the other hand, the separation of acoustic eigenfrequencies $\Delta v_{1}^{(a c)}$ is usually much smaller than the width of optical mode eigenfrequencies, which, e.g., is equal to $200 \mathrm{KHz}$ for optical Q-factor $Q^{(o p)}=10^{8}$ and frequency $200 \mathrm{THz}$.
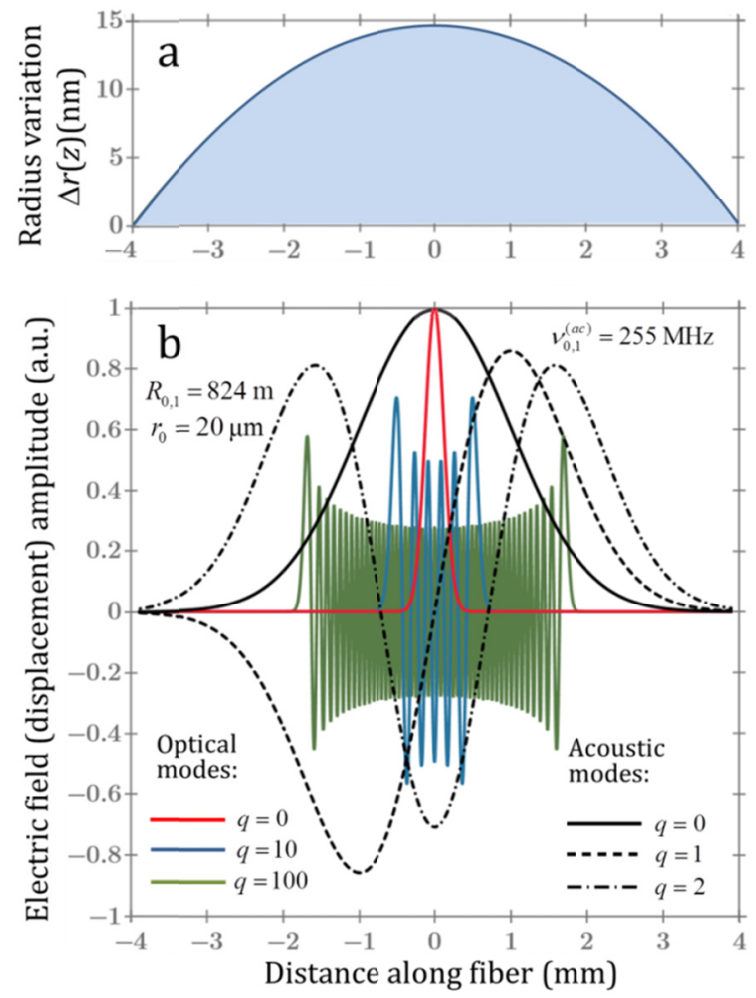

Fig. 3. (a) - Radius variation of parabolic bottle resonator with axial radius $R_{0,1}=824 \mathrm{~m}$. The acoustic cutoff frequency $v_{0,1}^{(a c)}=255 \mathrm{MHz}$ of this resonator is equal to the separation of its optical eigenfrequencies along the axial quantum number $q$. (b) - Axial distribution of optical and acoustic modes in the same resonator.

In summary, the theory of slow acoustic modes propagating in an optical fiber with nanoscale radius variation is developed and applied to comparative analysis of optical and acoustic bottle resonators. A new type of acoustic resonator, the neck-shaped antibottle resonator, is introduced. The future work consists in generalization of Eq. (3) (which, as shown above, is valid for both optical and acoustic modes) by taking into account nonlinear optomechanical interactions. If the optical back-action is weak and can be ignored, the effect of acoustic vibrations is described by one-dimensional non-stationary Schrödinger equation with timedependent potential proportional to the fiber radius variation $\Delta r(z)$ [24]. Taking advantage of this quantum-mechanical analogy, the phenomena known in non-stationary quantum mechanics can be demonstrated optomechanically. Examples include inelastic transitions in coupled quantum wells [25], the effect of absolute transparency of inelastic channel [26] which can be realized using the recently fabricated double quantum well structure [11], tunneling in an alternating field [27, 28], and others.

Funding. Royal Society (WM130110). Horizon 2020 (H2020EU.1.3.3, 691011).

Acknowledgment. The author acknowledges the Royal Society Wolfson Research Merit Award.

\section{References}

1. M. Sumetsky, Opt. Lett. 29, 8 (2004).

2. Y. Louyer, D. Meschede, and A. Rauschenbeutel, Phys. Rev. A 72, 031801(R) (2005).

3. M. Pöllinger and A. Rauschenbeutel, Opt. Express 18, 17764 (2010).

4. A. A. Savchenkov, A. B. Matsko, W. Liang, V. S. Ilchenko, D. Seidel and L. Maleki, Nat. Photon. 5, 293 (2011).

5. V. Dvoyrin and M. Sumetsky, Opt. Lett. 41, 5547 (2016).

6. M. N. M. Nasir, G. S. Murugan, and M. N. Zervas, JOSA B 33, 1963 (2016).

7. J. Volz, M. Scheucher, C. Junge and A. Rauschenbeutel, Nat. Photon. 8, 965 (2014).

8. M. Sumetsky, Phys. Rev. Lett. 111, 163901 (2013).

9. M. Asano, Y. Takeuchi, W. Chen, Ş. K. Özdemir, R. Ikuta, N. Imoto, L. Yang, and T. Yamamoto, Laser \& Photon. Rev. 10, 603 (2016).

10. M. Sumetsky, Nanophotonics 2, 393 (2013).

11. N. A. Toropov and M. Sumetsky, Opt. Lett. 41, 2278 (2016).

12. M. Aspelmeyer, T. J. Kippenberg, and F. Marquardt, Rev. Mod. Phys. 86, 1391 (2014)

13. A. W. Snyder and J. Love, Optical waveguide theory (Chapman and Hall 1983).

14. M. Sumetsky and J. M. Fini, Opt. Express 19, 26470 (2011).

15. L. D. Landau and E. M. Lifshitz, Theory of Elasticity (Pergamon Press, 1970).

16. These modes are of special importance for optomechanical applications since they can be excited by the axially symmetric radiation pressure of optical WGMs.

17. M. Onoe, H. D. McNiven, and R. D. Mindlin, Trans. ASME. J. Appl. Mech. 29, 729 (1962).

18. J. Zemanek Jr., J. Acoust. Soc. Am. 51, 265 (1972).

19. G. N. Greaves, A. L. Greer, R. S. Lakes, and T. Rouxel, Nature Mat. 10, 823 (2011).

20. M. Fukuhara, A. Sanpei, and K. Shibuki, J. Mat. Sci. 32, 1207 (1997).

21. X. Sun, K. Y. Fong, C. Xiong, W. H. P. Pernice, and H. X. Tang, Opt. Express 19, 22316 (2011).

22. R. O. Pohl, X. Liu, and E. Thompson, Rev. Mod. Phys. 74, 991 (2002).

23. A. D. Fefferman, R. O. Pohl, A. T. Zehnder, and J. M. Parpia, Phys. Rev. Lett. 100, 195501 (2008).

24. M. Sumetsky, Sci. Rep. 5, 18569 (2015).

25. S. Kohler, J. Lehmann, P. Hänggi, Phys. Rep. 406, 379 (2005).

26. M. Yu. Sumetsky and M. L. Felshtyn, JETP Lett. 53, 24 (1991).

27. M. Yu. Sumetskii, Sov. Phys. JETP 62, (1985).

28. B.I. Ivlev and V.I. Mel'nikov, Sov. Phys. JETP 63, 1295 (1986). 


\section{References with titles}

1. M. Sumetsky, "Whispering-gallery-bottle microcavities: the threedimensional etalon," Opt. Lett. 29, 8 (2004).

2. Y. Louyer, D. Meschede, and A. Rauschenbeutel, "Tunable whisperinggallery-mode resonators for cavity quantum electrodynamics," Phys. Rev. A 72, 031801(R) (2005).

3. M. Pöllinger and A. Rauschenbeutel, "All-optical signal processing at ultralow powers in bottle microresonators using the Kerr effect," Opt. Express 18, 17764 (2010).

4. A. A. Savchenkov, A. B. Matsko, W. Liang, V. S. Ilchenko, D. Seidel and L. Maleki, "Kerr combs with selectable central frequency," Nat. Photon. 5, 293 (2011).

5. V. Dvoyrin and M. Sumetsky, "Bottle microresonator broadband and lowrepetition-rate frequency comb generator," Opt. Lett. 41, 5547 (2016).

6. M. N. M. Nasir, G. S. Murugan, and M. N. Zervas "Spectral cleaning and output modal transformations in whispering-gallery-mode microresonators," JOSA B 33, 1963 (2016).

7. J. Volz, M. Scheucher, C. Junge and A. Rauschenbeutel, "Nonlinear $\pi$ phase shift for single fibre-guided photons interacting with a single resonatorenhanced atom," Nat. Photon. 8, 965 (2014).

8. M. Sumetsky, "Delay of Light in an Optical Bottle Resonator with Nanoscale Radius Variation: Dispersionless, Broadband, and Low Loss," Phys. Rev. Lett. 111, 163901 (2013).

9. M. Asano, Y. Takeuchi, W. Chen, Ş. K. Özdemir, R. Ikuta, N. Imoto, L. Yang, and T. Yamamoto "Observation of optomechanical coupling in a microbottle resonator," Laser \& Photon. Rev. 10, 603 (2016).

10. M. Sumetsky, "Nanophotonics of optical fibers," Nanophotonics 2, 393 (2013).

11. N. A. Toropov and M. Sumetsky, "Permanent matching of coupled optical bottle resonators with better than $0.16 \mathrm{GHz}$ precision," Opt. Lett. 41, 2278 (2016).

12. M. Aspelmeyer, T. J. Kippenberg, and F. Marquardt, "Cavity optomechanics," Rev. Mod. Phys. 86, 1391 (2014).

13. A. W. Snyder and J. Love, Optical waveguide theory (Chapman and Hall 1983).

14. M. Sumetsky and J.M. Fini, "Surface nanoscale axial photonics," Opt. Express 19, 26470 (2011).

15. L.D. Landau and E.M. Lifshitz, Theory of Elasticity (Pergamon Press, 1970).

16. These modes are of special importance for optomechanical applications since they can be excited by the axially symmetric radiation pressure of optical WGMs.

17. M. Onoe, H. D. McNiven, and R. D. Mindlin, "Dispersion of axially symmetric waves in elastic rods, " Trans. ASME. J. Appl. Mech. 29, 729 (1962).

18. J. Zemanek Jr., "An experimental and theoretical investigation of elastic wave propagation in a cylinder," J. Acoust. Soc. Am. 51, 265 (1972).

19. G. N. Greaves, A. L. Greer, R. S. Lakes, and T. Rouxel, “Poisson's ratio and modern materials," Nature Mat. 10, 823 (2011).

20. M. Fukuhara, A. Sanpei, and K. Shibuki, "Low temperature-elastic moduli, Debye temperature and internal dilational and shear frictions of fused quartz," J. Mat. Sci. 32, 1207 (1997).

21. R. O. Pohl, X. Liu, and E. Thompson, "Low-temperature thermal conductivity and acoustic attenuation in amorphous solids," Rev. Mod. Phys. 74, 991 (2002).

22. A. D. Fefferman, R. O. Pohl, A. T. Zehnder, and J. M. Parpia, "Acoustic properties of amorphous silica between 1 and 500 mK," Phys. Rev. Lett. 100, 195501 (2008).

23. X. Sun, K. Y. Fong, C. Xiong, W. H. P. Pernice, and H. X. Tang, "GHz optomechanical resonators with high mechanical $Q$ factor in air," Opt. Express 19, 22316 (2011).

24. M. Sumetsky, "Microscopic optical buffering in a harmonic potential,"Sci. Rep. 5, 18569 (2015).

25. S. Kohler, J. Lehmann, P. Hänggi, "Driven quantum transport on the nanoscale," Phys. Rep. 406, 379 (2005).
26. M. Yu. Sumetsky and M. L. Felshtyn, “Absolute transparency of inelastic channel and the photovoltaic effect in the resonance tunneling through the two-well heterojunction" JETP Lett. 53, 24 (1991).

27. M. Yu. Sumetskii, "Inelastic tunneling by a particle interacting with oscillations," Sov. Phys. JETP 62, (1985).

28. B.I. Ivlev and V.I. Mel'nikov, "Semiclassical processes in a high-frequency field," Sov. Phys. JETP 63, 1295 (1986). 